\title{
Experiences and Preferences of Opioid-Use- Disorder Patients Who Switched from Brand to Generic Buprenorphine/Naloxone Films: A Case Series
}

\author{
Erica Cedeño' \\ Ambar Cruz' \\ Joaris Cortés ${ }^{2}$ \\ Kyle Melin (iD) \\ Luis Roman ${ }^{3}$ \\ Angel Gonzalez ${ }^{3}$ \\ Jorge Duconge $\mathbb{1 D}^{4}$ \\ Darlene Santiago (iD ${ }^{4}$ \\ 'Department of Pharmacy Practice, \\ School of Pharmacy, Medical Sciences \\ Campus, University of Puerto Rico, \\ San Juan, Puerto Rico; ${ }^{2}$ Department of \\ Chemistry, Rio Piedras Campus, \\ University of Puerto Rico, San Juan, \\ Puerto Rico; ${ }^{3}$ SANOS Corporation, \\ Caguas, Puerto Rico; ${ }^{4}$ Department of \\ Pharmaceutical Sciences, School of \\ Pharmacy, Medical Sciences Campus, \\ University of Puerto Rico, San Juan, \\ Puerto Rico
}

Purpose: Differences in the suppression of withdrawal symptoms have been observed in opioid-use-disorder (OUD) patients who were switched from Suboxone (the brand name of buprenorphine/naloxone sublingual films) to either 1 of 2 generic versions. These descriptive observations evidence the need to further assess the use of these generics and its impact on the adherence to and outcomes of OUD treatments. The objective of this case series was to describe patient and provider experiences, perceptions, and preferences when said patients were abruptly switched from Suboxone to one of the generic versions manufactured by Sandoz or Alvogen.

Patients and Methods: A retrospective chart review of 24 Suboxone-maintained OUD patients from a single clinic who were forced to switch to a generic was performed to collect withdrawal and craving symptoms that occurred after the switch, as well as toxicology results and changes in dose (documented by the provider).

Results: The medical records of $9(37.5 \%)$ of the 24 patients showed that they were suffering from withdrawal symptoms and/or cravings, had had their doses adjusted, and/or had had a positive urine toxicology screen. All 9 subjects communicated a preference for the brand formulation over that of either of the generic versions; few expressed a preference for one generic formulation over the other. None of patients were able to switch back to the brand formulation, nor were any of them able to choose the generic that worked best for them. Insomnia, muscle pain, and gooseflesh skin were the most common withdrawal symptoms reported by the patients using the generics. Better outcomes were observed in patients who received a buprenorphine dose increase $(2 \mathrm{mg})$ to suppress the withdrawal symptoms experienced while using the generics.

Conclusion: Our study serves as a reference to prescribers regarding approaches (eg, a small dose adjustment) that may potentially encourage OUD treatment adherence and even improve outcomes in patients who appear to be decompensating after the brand-togeneric switch.

Keywords: buprenorphine, buprenorphine generics, opioid-use disorder, withdrawal symptoms, relapse, cravings, brand-to-generic switch, Suboxone

\section{Introduction}

Buprenorphine is safely and effectively prescribed in Medication-Assisted Treatment programs for patients with opioid-use disorder (OUD). ${ }^{1}$ It is commercially available as a single-ingredient sublingual (SL) tablet and as a combination drug with naloxone (as
Correspondence: Darlene Santiago

Department of Pharmaceutical Sciences, School of Pharmacy, University of Puerto Rico, Medical Sciences Campus, PO Box 365067, San Juan, 00936-5067, Puerto Rico

Tel +I 787-758-2525 ext. 5313

Email darlene.santiago@upr.edu 
SL and buccal films and as SL tablets). ${ }^{2}$ Suboxone (the brand name formulation of buprenorphine/naloxone) has been widely prescribed and has, according to reports, led to generally positive outcomes in terms of efficacy and safety., When the Food and Drug Administration (FDA) approved the generic versions of the Suboxone (buprenorphine/naloxone) films in $2018,{ }^{5}$ although these generics were considered to perform the same way as the brand medication did (same high quality, strength, purity, and stability), ${ }^{6}$ the unique opportunity to describe patient and provider experiences of both the brand-name and generic versions of this medication arose. High Addiction Severity Index scores and the use of higher-than-normally-prescribed daily doses have been associated with a patient preference for the Suboxone brandname formulation over the generic buprenorphine tablets. ${ }^{7}$ Additionally, a recent short report, although inconclusive, indicated that some patients experienced withdrawal symptoms when abruptly switched from Suboxone to the generic versions of the films. ${ }^{8}$ Most of the reports that have described the brand-to-generic switch (between films and tablets) have relied strictly on inconclusive clinical observations; a study focusing on the clinical manifestations in maintenance-phase patients who have been abruptly switched from brand-name Suboxone to generic versions of the film is, to our knowledge, lacking. The objective of this case series study was to describe the experiences and perceptions of patients and providers when those patients were abruptly switched - due to administrative decisions of the medical insurance carriers - from Suboxone to the generic versions manufactured by Sandoz and Alvogen. This is the first report to describe cases in a Suboxone-maintained OUD patient population, which cases consisted of patients who were switched to generic formulations, and their experiences of clinical deterioration and/or decompensation, with those experiences being recorded in their medical records by their providers.

\section{Materials and Methods Study Design}

We performed an observational retrospective case series study amongst an OUD patient population of a single clinic to describe the experiences of buprenorphine-maintained OUD patients who were forced to switch from the brandname buprenorphine-naloxone SL film (Suboxone) to 1 or both of the available generics (produced by from Sandoz and Alvogen). To that end, we also wanted to depict the experiences of those reporting withdrawal symptoms and cravings after the mandatory switch. The electronic records were reviewed to identify patients that experienced symptoms of withdrawal and/or cravings, as observed and documented by the provider, and any associated dose adjustments and/or positive results for illicit drug use that were noted after the switch was made. The mandatory brand-to-generic switch occurred after a change was made to the formulary of the government medical insurance in April 2019. This change forced all the pertinent insured individuals to switch to 1 (or both) of the generic versions of Suboxone; affected were both OUD patients considered to be stable in their current treatment and new patients being initiated into treatment.

The patients presented herein represent a convenience sample from a single OUD clinic in Puerto Rico. To protect the confidentiality of the recruited subjects, the name of the clinic has been kept anonymous. This study was approved prior to its commencement by the Institutional Review Board of the University of Puerto Rico Medical Sciences Campus (FAWA00005561, protocol B1080220; approved on August 31, 2020), and in accordance with the Declaration of Helsinki. The study was designed in such a way as to keep its subjects anonymous; thus, no identifying information was collected. For the study, verbal consent was obtained from all the participants for the collection and dissemination of their data, these data were collected using IRB-approved forms, no HIPAA identifiers were collected, and only that clinical data related to each patient's OUD was recorded. The only demographic variables that were collected were age and sex. Our study received a written authorization from the IRB to collect and disseminate health-related information after first obtaining verbal consent from the subjects. The authorization was granted because our planned use of IRB-approved forms to collect the data meant that there would be little or no risk that the subjects would experience a loss of privacy. In addition, no identifiers would be collected and what information was to be collected would be destroyed after the study had come to a close. Finally, there was no intention to re-use or disclose (except as required by law and/or for research and dissemination purposes) the collected health information. All the research staff completed HIPAA training before the study started to recruit patients. Written informed consent to publish these nice cases details was provided by all nine participants.

\section{Inclusion/Exclusion Criteria}

The inclusion criteria were that a participant must be 21 years of age or older, have been diagnosed with OUD (according to the Diagnostic and Statistical Manual IV), be receiving a maintenance dose of Suboxone film (steady state), and have been forced (in April 2019) to switch to 1 
or both of the FDA-approved generic versions manufactured by Sandoz and Alvogen. Patients who - due to the insurance formulary change - were switched to the SL tablet or to another formulation other than the SL film were excluded.

\section{Measured Outcomes}

The selected cases included individuals who, after the switch, had documented (by the provider) symptoms of withdrawal and cravings and associated dose adjustments and/or positive results for illicit drugs (Table 1).

\section{Results}

The clinic from which the cases were culled was providing care for a total of 92 patients in April 2019: 47 were OUD patients, and of those 47, only 24 were prescribed SL buprenorphine films, were not induced into treatment, and received no dose titrations in the months prior to the formulation switch. These 24 patients were approached, and all of them provided consent to participate in the study. A retrospective chart review of the electronic medical records from January through October 2019 of these 24 patients was performed. Opioid-use-disorder treatment-specific information, such as the presence of withdrawal symptoms, cravings for opioids, dose adjustments of the film, urine toxicology reports, and information from the physician notes, was documented in each patient's chart. Additionally, demographic (age, gender, marital status, race, ethnicity, town of residence, and employment status) and general clinical (medical, family, social, substance use and medication history, and presence of allergies) data were collected.

Based on the information acquired from the chart reviews, 9 patients $(37.5 \%)$ out of the 24 reported having withdrawal symptoms and/or cravings, either or both of which were accompanied by a dose adjustment and/or a positive urine toxicology result. Below are descriptions of these patients.

\section{Case Presentations Case I}

This participant was a 37-year-old, unemployed, single male with a past medical history of severe obesity and hypertension and a history of using tobacco, marijuana, oxycodone/acetaminophen, and tramadol (the last of

Table I Variables Analyzed to Determine the Outcome After the Brand-to-Generic Switch of Buprenorphine/Naloxone Sublingual Films

\begin{tabular}{|c|c|c|c|}
\hline Dose Adjustment & Positive Urine Toxicology Test & Withdrawal Symptoms & Cravings for Opioids \\
\hline $\begin{array}{l}\text { Dose changes in milligrams } \\
\text { of buprenorphine-naloxone } \\
\text { film during routine } \\
\text { appointments: } \\
2 \mathrm{mg} / 0.5 \mathrm{mg} \\
4 \mathrm{mg} / \mathrm{l} \mathrm{mg} \\
8 \mathrm{mg} / 2 \mathrm{mg} \\
12 \mathrm{mg} / 3 \mathrm{mg}\end{array}$ & $\begin{array}{l}\text { Any positive result(s) for illicit or } \\
\text { prescribed drugs. The toxicology panel } \\
\text { used in the clinic included the following } \\
\text { substances: } \\
\text { I I-panel drug test*: } \\
\text {-Amphetamines } \\
\text {-Benzodiazepines } \\
\text {-Cocaine } \\
\text {-Methamphetamines } \\
\text {-Methadone } \\
\text {-Opiates** } \\
\text {-Oxycodone } \\
\text {-Barbituates } \\
\text {-Buprenorphine } \\
\text {-PCP } \\
\text { - THC } \\
\text { Individual strips**: } \\
\text {-Fentanyl } \\
\text { Tramadol (only when required, based } \\
\text { on patient's addiction or history of } \\
\text { consumption) }\end{array}$ & $\begin{array}{l}\text { Symptoms produced by abrupt cessation, } \\
\text { rapid dose reduction, decreasing blood } \\
\text { levels of the drug, and/or administration of } \\
\text { an antagonist. Reported at least I of the } \\
\text { following: } \\
\text { - Restlessness } \\
\text { - Irritability } \\
\text { - Increased sensitivity to pain } \\
\text { - Nausea } \\
\text { - Vomiting } \\
\text { - Diarrhea } \\
\text { - Cramps } \\
\text { - Lacrimation } \\
\text { - Rhinorrhea } \\
\text { - Muscle aches } \\
\text { - Insomnia } \\
\text { - Anxiety } \\
\text { - Dysphoria } \\
\text { - Yawning } \\
\text { - Sweating } \\
\text { - Piloerection } \\
\text { - Fever }\end{array}$ & $\begin{array}{l}\text { The desire, want, urge, or } \\
\text { need to use opioids, } \\
\text { particularly illicit ones, } \\
\text { such as heroin. }\end{array}$ \\
\hline
\end{tabular}

Notes: *The II-panel drug test measures the presence of these different drugs in a urine sample. It discriminates between opiates and opioids such as oxycodone. **Opiates are chemical compounds extracted or refined from natural plant matter; examples of opiates measured in this test include heroin and morphine. ***Tests not included in the II-panel drug test. The strip is dipped in the urine sample to measure consumption, whenever there is a history of fentanyl and/or tramadol abuse. 
which had not been prescribed). His medication history included enalapril and doxepin.

This patient started OUD treatment with buprenorphine tablets in the fall of 2018; however, early in 2019, he was switched to Suboxone films after communicating a desire for the film and was prescribed $3 \mathrm{SL}$ films $(8 \mathrm{mg} / 2 \mathrm{mg}$, each; $24 \mathrm{mg}$ BUP, total), daily, in March 2019. The patient reported high satisfaction with this formulation. The patient faced personal family struggles and situations in the months prior to the brand-to-generic switch but had-after said switch - no positive toxicology results for illicit drug use; his record showed no dosage adjustments of buprenorphine. No adverse effects were documented previous to the brandto-generic formulation switch, with only 1 positive result for cannabis during March 2019.

In June 2019 ( 2 months after the brand-to-generic formulation switch), the patient's record showed that his physician added 1 additional SL film $(2 \mathrm{mg} / 0.5 \mathrm{mg})$ to his usual maintenance dose of $3 \mathrm{SL}$ films $(8 \mathrm{mg} / 2 \mathrm{mg}$, each; $26 \mathrm{mg}$ BUP, total), daily. The medical record showed that instructions were given to the patient to use this additional dose at night. This came after the patient reported, in that same month, having experienced withdrawal symptoms; no additional reports on the presence of opioid cravings were found in his record. In his next visit (July 2019), the patient reported having experienced differences with this new formulation (the need for additional buprenorphine films before the next scheduled dose due to the earlier offset of the medication). From this month until the end of our observations (October 2019), no withdrawal symptoms were reported in the patient's record. In August 2019, the patient said that the generic buprenorphine-naloxone films - which he described as the "white films"-were "weaker" (less effective) than the "original" (brand name) ones were; he persisted in expressing how different the generic ones "feel." The urine toxicology tests were negative for fentanyl and other opioids after the switch from the brand to the generic formulation.

\section{Case 2}

This participant was a 55-year-old, unemployed, single male who was experiencing homelessness at the time of the study and had a past medical history that included chronic viral hepatitis $\mathrm{C}$, persistent mood [affective] disorder and major depressive disorder, and a Benadryl allergy. Additionally, the patient had a history of using nicotine, heroin, alprazolam, and cocaine and had been diagnosed with a dependence disorder with respect to these drugs. Trazodone and sertraline were the only prescription medicines that the patient's record showed. In addition, the provider's notes in that record showed that the patient was struggling with depression related to the deaths of some close family members.

The patient started OUD treatment with buprenorphine-naloxone in January of 2017. The record showed that throughout his OUD treatment, the patient complained of withdrawal symptoms (anxiety and insomnia), and although no instances of cravings were documented by the provider, the patient's illicit drug use (toxicology) indicated that the patient experienced cravings, with the medical record showing various dose adjustments to address them. In the months prior to April 2019, he was prescribed 3 SL films $(8 \mathrm{mg} / 2 \mathrm{mg}$, each; $24 \mathrm{mg}$ BUP, total), daily. Although adherent to his buprenorphine pharmacotherapy (positive urinalysis for buprenorphine) and his monthly appointments, this patient showed evidence of illicit drug use (cocaine and opioids [including fentanyl]). After April 2019 (date of the switch from brand to generic), this patient missed 2 appointments, 1 in the month of May 2019 and 1 in the month of July 2019. The patient continued using illicit substances (opioids: heroin and fentanyl). In June 2019 ( 2 months after the formulation switch) the patient complained that the generic SL film was ineffective. The patient's toxicology results for this month were positive for fentanyl. In July and August, the patient reported needing more films to keep himself "stable"; he had taken additional films and thus depleted his monthly supply and, as a result, had run out of medication before his next scheduled refill. Both in July and August, his toxicology screens were positive for opioids - fentanyl - and cocaine and negative for buprenorphine. The patient admitted to using heroin and fentanyl, which was confirmed with positive urine toxicology results after tests for opioids (July to October), specifically for fentanyl (June to October), and for cocaine (July, August, and October). This patient had resumed using illicit drugs, but no withdrawal symptoms or cravings for opioids were reported in his medical record, and no dose adjustments were made by his provider.

\section{Case 3}

This 50-year-old, single male had a past medical history of obesity, anemia, mental/behavioral problems, and hypertension, and he had a penicillin allergy. The patient had a social history of abusing alcohol and other drugs (the chart review did not reveal the types of drugs). His clinical 
record indicated that he had prescriptions for tamsulosin and trazodone.

This patient began treatment with buprenorphinenaloxone back in January 2014 (prior to starting treatment, the patient reported illegally purchasing Suboxone on the street). During the following years, the patient was in and out of treatment. According to his record, in 2018, the patient had various dose adjustments because of his having experienced symptoms of withdrawal and cravings and having tested positive for illicit drugs (fentanyl, heroin, oxycodone). The record also showed a fentanyl overdose right after a decrease in his Suboxone dose. The patient identified gooseflesh skin as the major withdrawal symptom that needed to be suppressed. Although stating that most of these symptoms were suppressed with his prescribed 1.5 SL films (8 mg/2 mg, each; $12 \mathrm{mg}$ BUP, total), daily, he expressed a desire for an increase to 2 SL films (8 mg/2 mg; $16 \mathrm{mg}$ BUP, total), daily, for complete suppression. No drug cravings or withdrawal symptoms were reported after this last dose increase. In the months prior to the formulation switch, his toxicology tests came back positive for opioids (February and March 2019) and negative for buprenorphine (February 2019).

After the formulation switch, the patient did not receive any dose adjustments. And even though the medical record of this patient did not show extensive documentation throughout in terms of observed or communicated withdrawal symptoms or drug cravings, this patient continued having positive urine results for opioids (May, August, and October); fentanyl-positive results were documented during June, July, August, and October.

\section{Case 4}

This was a 53-year-old, unemployed, single female with a past medical history of heart disease, chronic viral hepatitis, overweight, major depressive disorder, chronic migraine, polyneuropathy, hypertension, calcific tendonitis of the left shoulder, and chondrocostal junction syndrome. Nicotine and alcohol abuse were part of her substance-use history, which included a diagnosis of nicotine dependence and alcoholism. She also had a diagnosis of addiction to heroin and cocaine. The patient used the following medications: sertraline, trazodone, risperidone, clonazepam, lisinopril, aspirin, nabumetone, ketorolac, triamcinolone, salsalate, promethazine, and hydroxyzine pamoate. Before starting treatment with buprenorphine-naloxone, she had been on methadone for 23 years.
The patient began OUD treatment with buprenorphinenaloxone films in January 2018. The presence of cravings was noted in February 2018, and after she admitted to both fentanyl use and instances of self-harming, her dose was increased from 1 to $2 \mathrm{SL}$ films $(8 \mathrm{mg} / 2 \mathrm{mg}$, each; $16 \mathrm{mg}$ BUP, total), daily. She continued with a dose of 2 SL films (8 mg/2 mg, each, daily) until April 2019. Her toxicology reports were negative for opioids and fentanyl from February to April 2019 (toxicology results for January were not available). In February 2019, a urinalysis was negative for buprenorphine.

After switching from the brand to the generic formulation of buprenorphine-naloxone in April 2019, there was no documentation about the patient regarding the switch, the presence of withdrawal symptoms, or cravings for opioids during our observation period. In May 2019, the patient was prescribed $1 \mathrm{SL}$ film $(2 \mathrm{mg} / 0.5 \mathrm{mg})$, daily, in addition to her previous prescription, up until the last observation (18 mg BUP, total). The toxicology findings for this participant varied: She was positive for buprenorphine from May to October 2019, indicating her adherence to the treatment. She was positive for opioids from the end of September to mid-October 2019 and positive for fentanyl from June to mid-October 2019. She was positive for cocaine at the beginning of October 2019. Additionally, she was positive for benzodiazepines from May to October 2019. However, the patient had prescriptions for clonazepam from the clinic in February, March, May, August, and October of 2019.

\section{Case 5}

A 58-year-old, female patient, single and unemployed, had a past medical history that included bipolar disorder, major depressive disorder, and HIV. Past alcohol and cocaine use and current tobacco use were part of her social history, but in addition to her medications for OUD, her clinical record did not provide additional information about her history (if any) of taking prescription medications.

In June 2018, the patient started OUD treatment with 2 SL films, daily, of buprenorphine-naloxone $(8 \mathrm{mg} / 2 \mathrm{mg}$, each;16 mg BUP, total); no drug cravings or withdrawal symptoms were reported at this time. By the end of that year, in December 2018, her physician added $1 \mathrm{SL}$ film, daily ( $2 \mathrm{mg} / 0.5 \mathrm{mg}$ ), after the patient reported having experienced cravings, a loss of enthusiasm, and insomnia. In January 2019, before the switch, she was classified as stable, with 2 SL films ( $8 \mathrm{mg} / 2 \mathrm{mg}$, each), daily, in addition to $1 \mathrm{SL}$ film (2 mg/0.5 mg), daily, for a total of $18 \mathrm{mg}$ 
BUP; the record showed that she expressed feeling "strong" at this dose. She did not have a positive urine toxicology screen previous to the switch.

In July 2019, 3 months after the switch, the patient noticed differences in the effectiveness of the generic versions of Suboxone, with, according to her, the Sandoz formulation being more effective than Alvogen's, but with both formulations being less effective than that of Suboxone. The clinical record showed that in August she had relapsed into illicit drug use. The physician wrote in her record that the patient felt ambivalent about the new film and that in order to "feel better," she was taking additional doses, thereby depleting her monthly supply. Urine screening was also positive for fentanyl that month (August). As a result, the physician added 1 additional SL film (8 mg/2 mg; $24 \mathrm{mg}$ BUP, total), per day; the $2 \mathrm{mg}$ / $0.5 \mathrm{mg}$ SL film was not prescribed. This last dose was maintained through our last observation in October 2019. The patient's toxicology tests were negative for other opioids following the increase, and the medical record did not include any more reports of withdrawal symptoms from that point on.

\section{Case 6}

This case was of a 38-year-old, unemployed, single male with bipolar disorder, major depressive disorder, and generalized anxiety disorder. His substance history included the abuse of alcohol, tobacco, marijuana, and heroin. Further, his medication history included quetiapine, cortisol cream, and medicinal cannabis.

He started OUD treatment with buprenorphine-naloxone films in October 2018 and stabilized with 2 SL films ( $8 \mathrm{mg} / 2 \mathrm{mg}$, each) per day in November 2018 (stable in maintenance; $16 \mathrm{mg}$ BUP, total), with no withdrawal symptoms reported. The patient communicated that he was satisfied with the pharmacotherapy and continued with the regimen. In the months prior to the switch, the patient had a difficult personal situation and reported having experienced cravings for drugs, cravings that led to his use of illegal substances in March and April 2019 (cocaine, benzodiazepines, and opioids); he missed 1 routine appointment. The record showed that the patient purchased Suboxone, illegally, on the street, presumably because of his having missed his last appointment. In April 2019, 1 SL film (2 mg/0.5 mg), daily, was added to his then daily dose (18 mg BUP, total); this increase was made after the patient reported having experienced cravings and feelings of anxiety. In May 2019, the patient continued reporting daily anxiety and cravings that manifested as body aches, sweating, and headaches, all of which manifestations went away after he took the buprenorphine SL films. The patient also reported that as the day progressed, the craving to use drugs increased, peaking at around 2 to $3 \mathrm{PM}$. In that same month (May of 2019) the patient's urinalysis was positive for illicit opioids and benzodiazepines. At this point, the patient's dose was increased to $3 \mathrm{SL}$ films ( $8 \mathrm{mg} / 2 \mathrm{mg}$, each; $24 \mathrm{mg}$ BUP, total), daily; the patient reported feeling much better with the new dose. The following month (June 2019), the patient's urinalysis was positive for cocaine.

Through the next couple of months (June-August 2019), when asked about the generic films, the patient said that the Sandoz films gave him headaches, constipation, sweating, and hot flashes and that the Alvogen films were less effective compared to those from Sandoz at suppressing withdrawal symptoms.

\section{Case 7}

A 49-year-old, unemployed, single male who was living with his mother at the time of the study had a past medical history that included bipolar disorder, persistent mood [affective] disorder, anxiety disorder, chlamydia, and hepatitis C. His social history included the consumption of alcohol, tobacco, coffee, and other drugs (his chart did not specify which other drugs he used). According to his clinical record, he had prescriptions for trazodone, hydroxyzine, and nabumetone during the time under study.

He started OUD treatment with 2 SL films, daily, of buprenorphine-naloxone ( $8 \mathrm{mg} / 2 \mathrm{mg}$, each; $16 \mathrm{mg}$ BUP, total) in October 2017 and by November of that year was considered stable at that dose. However, in the years that followed, several hospitalizations and personal emergencies occurred that impacted his treatment for OUD. In December 2017, he had to be hospitalized in a mental health institution due to depression and anxiety, combined with financial and housing problems after the passing of Hurricane Maria. One year later (December 2018), the patient reportedly had to be revived with Naloxone when he overdosed after being temporarily incarcerated. In January 2019, the patient resumed his treatment with the same dose (16 mg BUP, total), and his record showed that during this appointment, he complained that his current prescription was not sufficient to manage his symptoms and that he had illicitly purchased Suboxone on the street. His urine toxicology tests were negative for opioids, cocaine, and fentanyl. 
In May 2019 (one month after the switch), the presence of drug cravings was noted in his clinical record, with these increasing as the months passed. These cravings (accompanied by significant gooseflesh and muscle aches, as reported by the patient) led to a dose change in August 2019. The patient tested positive for fentanyl and cocaine in June 2019 and reported that he had used heroin that month, as well. On August 14, the patient went from 2 SL films ( $8 \mathrm{mg} / 2 \mathrm{mg}$, each; $16 \mathrm{mg}$ BUP, total), daily, to 2 SL films ( $8 \mathrm{mg} / 2 \mathrm{mg}$, each), daily, combined with a single, daily $2 \mathrm{mg} / 0.5 \mathrm{mg} \mathrm{SL} \mathrm{film}$, for a total of $18 \mathrm{mg} \mathrm{BUP}$; at this time, he was going to bi-weekly appointments. On August 27, his next appointment, the physician further increased his dose to $2.5 \mathrm{SL}$ films $(8 \mathrm{mg} / 2 \mathrm{mg}$, each; $20 \mathrm{mg}$ BUP, total) per day. In this new dose the patient reported feeling better. In October 2019, the patient complained again of having back aches and shivers in the morning but these resolved with the morning film.

The patient did not express perceiving significant differences between Suboxone and the generic versions; however, in August 2019, when comparing the 2 generic films he had being using, he indicated that the current films did not provide the same effect in terms of "energy" as did the previous generic ones. He detailed that when feeling "down" and taking a film, the previous generic ones would lift him up, but the current ones did not.

\section{Case 8}

A 45-year-old, unemployed, single male, with a social history including alcohol consumption and tobacco, heroin, and medicinal cannabis use. His medication history included clonazepam, sertraline, temazepam, and ranitidine.

The patient started OUD treatment in September 2017 with SL buprenorphine/naloxone tabs and was switched to Suboxone films in November 2018, with 3 SL films ( $8 \mathrm{mg} /$ $2 \mathrm{mg}$, each; $24 \mathrm{mg}$ BUP, total), daily. The patient was considered stable at this dose, and also reporting having used medicinal cannabis. From January through April 2019, his urine toxicology results were positive for THC and negative for fentanyl, illicit opioids, and benzodiazepines.

The patient's record showed that after he was switched to the generics, he needed to take his daily dosage earlier in the morning than he had normally done when taking Suboxone; otherwise, he would experience body aches, would vomit, and/or would be irritable. No dose adjustments were reported after the transition (from the brand to the generic formulation), and the patient did not have any urine toxicology results that were positive for opioids, more specifically, for fentanyl, after said transition.

\section{Case 9}

Our last participant was a 43-year-old, homeless male who was unemployed and single at the time of the study. He had a medical history of hypertension and a substance-use history that included alcohol, tobacco (5 cigarettes, daily), heroin, and cocaine. In addition to the buprenorphinenaloxone SL films, the patient was taking trazodone, which had been prescribed at the clinic during the study period.

He started treatment with 1 SL film, daily, of buprenorphine-naloxone, $8 \mathrm{mg} / 2 \mathrm{mg}$, in September 2018, and in October, his dose was increased to $1.5 \mathrm{SL}$ films $(8 \mathrm{mg} /$ $2 \mathrm{mg}$, each; $12 \mathrm{mg} \mathrm{BUP,} \mathrm{total),} \mathrm{daily,} \mathrm{after} \mathrm{reporting} \mathrm{drug}$ cravings and having used illicit drugs. In his next appointment, in October, he was classified by his provider as being underdosed. He reported having had drug cravings and withdrawal and other undesirable symptoms, so his dose was further increased to $2 \mathrm{SL}$ films $(8 \mathrm{mg} / 2 \mathrm{mg}$, each; $16 \mathrm{mg}$ BUP, total), daily. By the end of the year (December 2018), the physician classified him as stable, with no withdrawal symptoms being reported afterwards. He continued this stable regimen through April 2019. Prior to the change in formulation, the patient's urine toxicology results were negative for opioids including fentanyl (January, March, and April, with no report available for February).

Following the switch to the generic formulation of Suboxone in May 2019, the patient reported only 1 withdrawal symptom, insomnia. He reported waking up during the night due to discomfort, which was alleviated only when he used additional doses of Suboxone, to which the patient reported having illegal access on the street. The patient also reported that when taking trazodone at night (as prescribed), his symptoms were alleviated; however, he discontinued its use due to the side effect of dry mouth. In June 2019, the patient reported having had cravings for opioids, stating that he had had a "bad moment," so he had to use illicit drugs. In May 2019, his dose of buprenorphine-naloxone was increased from $2 \mathrm{SL}$ films $(8 \mathrm{mg} /$ $2 \mathrm{mg}$, each; $16 \mathrm{mg}$ BUP, total), daily, to $2.5 \mathrm{SL}$ films (20 mg BUP, total), daily; later in June 2019, a final dose adjustment was made, increasing his daily intake to $3 \mathrm{SL}$ films (8 mg/2 mg, each; $24 \mathrm{mg}$ BUP, total). 


\section{Discussion}

Generic formulations of Suboxone SL films were approved by the FDA in June of 2018, based upon bioequivalence studies. ${ }^{5}$ Although these generic versions were certified to be bioequivalent to Suboxone, a potential exists for unexpected effects associated with the sudden switch from the brand-name version to 1 or both of the generic ones, in a given individual, which effects may become apparent only after the clinical use of said generics in and by the general population. Patients experiencing withdrawal symptoms when switched from the brand to the genetic formulations of buprenorphine (film and/or tablet) has been reported $;^{7-10}$ however, this is the first study to individually describe 1) the brand-to-generic switch of buprenorphine/naloxone SL films (Suboxone to 1 or both of the generic formulations manufactured by Sandoz and Alvogen, 2) all the Suboxone-maintained OUD patients from a single MAT clinic, and 3) patients' individual experiences and preferences as documented in their medical records by their providers. This study is particularly important as it elucidated the providers' approaches to successfully restabilizing patients who were not able to go back to their previous brand-name formulation. Important observations are discussed, as are future recommendations for OUD clinicians and providers alike, said recommendations being based on the clinical experiences presented in this article.

The patients had their specific preferences regarding the different formulations - brand-name over generics, one generic over another, and so on-the detailing of which is an important element of this study. Although this information was not available for every patient, such specifics regarding patient preferences as we were able to determine is relevant to and important for OUD clinical practices that are using buprenorphine/naloxone films with their patient populations. Most patients expressed a preference for the brand-name formulation, and a few expressed a preference for one generic formulation over the other. Due to the descriptive nature of our study, we were not able to examine any possible associations of patient preferences for the brand-name or generic formulations of buprenorphine with treatment outcomes. However, past studies (Binder et al) have associated the preference for the brand-name formulation over a generic with higher levels of addiction; that being the case, the brand-to-generic switch described herein may present a risk of relapse to patients with comparatively higher levels of dependency. ${ }^{7}$ None of the patients in this case series were able to receive the formulation they preferred; not one of them switched back to Suboxone films, and none were able to receive the generic version they identified as working the best for them. Contrarily, before this mandated brand-to-generic switch, a single Subutex-maintained (SL tablet) patient expressed a preference for the Suboxone film and was able to switch from the SL tablet to the film with no apparent resistance from the provider or insurance carrier, which switch resulted in the increased satisfaction and adherence of the patient. Better outcomes have been reported to occur when patients have the ability to switch back to a formulation that they feel works better for them, ${ }^{9}$ compared to mandated formulations that leave them feeling powerless and with no control over their therapy.

Although considered to be in a maintenance phase with Suboxone, some patients had cravings that led to illicit drug use before the switch (determined by toxicology results), which use continued after the switch. Illicit drug use while in the maintenance phase of OUD treatment may occur for various reasons that are not necessarily associated with buprenorphine's efficacy (or lack thereof); however, these illicit drug-use practices can potentially decrease a given prescriber's willingness to adjust (increase) his or her patient's buprenorphine dose when that patient appears to be decompensated. Illicit drug use while in treatment has been associated with lower retention rates, and this can be particularly dangerous when coupled with patient perceptions of their medication having lower efficacy. ${ }^{11,12}$ Most of the patients described in this study who were frequent illicit drug users before the switch were not prescribed a dose adjustment, even after having communicated that the generic films did not always have the same effect. The exact reason(s) that some patients received additional doses and others did not remains uncertain; however, the unwillingness of a specific provider to increase the dose in a patient with concurrent illicit drug use may have played a role. In addition, an insurance cap may have prevented dose increases in some patients after the switch, as patients that were prescribed $24 \mathrm{mg}$ of Suboxone did not receive dose increments when they began using the generic versions; such a cap would limit the ability of the provider to increase the dose of a patient who appears to be clinically decompensating.

This case series shows the clinical manifestation of withdrawal symptoms in OUD patients who, although considered stable in treatment, had negative outcomes when they were switched to generic versions of Suboxone. Insomnia, muscle pain, and gooseflesh skin were the 
withdrawal symptoms that were reported most often by patients after the switch to generic versions of Suboxone. All of the cases presented here were considered to be in the maintenance phase and were receiving a steady-state dose of Suboxone. The mechanisms leading to manifestations of withdrawal symptoms in these patients when switched to the generic versions of Suboxone require further clinical evaluation; however, these symptoms might suggest that there are small differences between Suboxone and its generic versions in terms of absorption and metabolism. We previously reported a 1-hour delay in reaching the maximum concentration of buprenorphine and the gradual onset of Sandoz-produced Suboxone generic in a case study that was able to measure the buprenorphine blood levels of a single patient while in steady state using Suboxone and, following that, while the patient was taking the Sandoz generic. ${ }^{10}$ Although the case series being described in this article was not designed to measure the buprenorphine blood levels of the participating patients, 2 of the cases presented here reported that while they experienced withdrawal symptoms taking a generic version of Suboxone, they also experienced differences in the offset of the generic compared to that of Suboxone, which offset precipitated in them a desire to take additional doses before it was time for their daily dose. Potential changes in the buprenorphine exposure of the generic versions of Suboxone may have impacted the saturation of the opioid receptors in the patients, causing them to feel sick earlier in the day after taking their daily dose. ${ }^{9}$ Several of the cases reported taking higher dosages than those indicated in their daily prescriptions, resulting in their running out of medication before their next refill; some of these patients resorted to illegally purchasing the films on the street. This misuse of buprenorphine/naloxone films (higher doses than prescribed) in comparison with other opioids, even buprenorphine tablets, presents not more than a low risk for diversion, mainly because of the difficulty of injecting them. Higher dosages than those prescribed when using generic buprenorphine have been previously reported in patients who had not previously used brand-name buprenorphine. ${ }^{7}$ Some patients in our study had their daily doses augmented by $2 \mathrm{mg}$. Patients taking the generic formulation and who received the increase, took the additional, small dose in the afternoon; by all reports, the patients felt better with the added dose. This 2-mg dose increase may have compensated for the reduced afternoon offset, which reduced offset may have precipitated withdrawal symptoms. These patients were restabilized and later expressed increased satisfaction with their treatment (suppression of withdrawal symptoms) and showed better outcomes compared to those who did not receive a dose increase.

This case series described the clinical experiences of OUD patients and their providers. Although important observations were made, there are some limitations to the study. A major limitation was that the study collected the perceived experiences of patients and providers without using validated scales to objectively assess withdrawal symptoms and cravings. Although patients who prefer the brand formulation over the generic ones might exaggerate their symptomatology, ${ }^{13}$ the described symptoms are still important and should be evaluated. That being the case, clinicians and providers of OUD patients should assess the withdrawal symptoms and cravings of those patients with the pertinent scales, especially when a given patient appears to be decompensating (when, for example, he or she has switched between the different formulations of the buprenorphine/naloxone films). Adopting this approach can aid the clinician/provider in making objective changes in the patient's pharmacotherapy, which changes might take the form of adjusting the patient's dosage. Another advantage of this particular strategy is that it gives the clinician/provider ammunition for fighting the patient's preconceived notions of the efficacy of the generic. Another limitation was that the study did not collected information on patients who did not show to be decompensating after the switch, it is important that proper socio demographics, substance use practices and medical history of this patient population be also described and compared.

This study also described how some patients could be restabilized after a small 2-mg dose increase (taken in the afternoon). Although this approach was not implemented with all the patients, it appeared to be effective in the patients that did receive this increase. The clinicians and providers of OUD patients should individually evaluate each patient who switches between the generic and brand-name formulations and consider a small dose adjustment (as a means of avoiding relapse and potential illicit drug use) to help the patient compensate for such clinically observed withdrawal symptoms as have been properly assessed using validated scales. Afterwards and when the patient is stable, a titration back to the previous dose before the switch might be considered. Further studies are required to accurately and objectively assess the broader impact of the brand-to-generic switch of buprenorphine/naloxone films; in the meantime, providers with OUD patients should closely monitor those 
patients when they are switched from the brand name to the generic buprenorphine/naloxone films.

\section{Conclusion}

This case series describes the clinical manifestations of a buprenorphine brand (Suboxone)-to-generic (manufactured by Sandoz and Alvogen) switch in a patient population visiting a single OUD clinic. Although small and descriptive, it serves as a reference for prescribers regarding approaches that may potentially help patients to maintain OUD treatment adherence and even improve outcomes when those patients appear to become destabilized after the brand-togeneric switch. Additionally, the observed preferences of many patients for formulations that were not provided to them due to administrative decisions made by their medical insurance carrier are also important information. Providers should be aware of these clinical manifestations and preferences when patients are switched; and these providers might want to consider a small dose adjustment to restabilize a given patient, if such an adjustment is clinically warranted. Further studies are necessary to better understand the impact of this kind of switch on the general OUD population.

\section{Acknowledgments}

This work was supported by the National Institutes of Health, award numbers HCTRECD R25MD007607, HiREC S21MD001830, and RCMI 8G12MD007600; Dr. Melin's time was supported by SC2 DA052099.

\section{Disclosure}

The authors report no conflicts of interest in this work.

\section{References}

1. US Department of Health and Human Services, Substance Abuse and Mental Health Services Administration Center for Substance Abuse Treatment. Clinical guidelines for the use of buprenorphine in the treatment of opioid addiction: a treatment improvement protocol (TIP 40); 2004.
2. Buprenorphine. Substance Abuse and Mental Health Services Administration (SAMHSA); May 14, 2021. Available from: https:// www.samhsa.gov/medication-assisted-treatment/medications-counsel ing-related-conditions/buprenorphine. Accessed June, 2021.

3. Comer S, Cunningham C, Fishman MJ, et al. American Society of Addiction Medicine (ASAM) National Practice Guideline for the use of medications in the treatment of addiction involving opioid use. $J$ Addict Med. 2015;9(5):358-367. doi:10.1097/ADM.0000000 000000166

4. National Academies of Sciences Engineering, Medicine. Medications for Opioid Use Disorder Save Lives. Leshner AI, Mancher M, editors. The National Academies Press (US); 2019. doi:10.17226/25310

5. FDA approves first generic versions of Suboxone sublingual film, which may increase access to treatment for opioid dependence. Food and Drug Administration; June 14, 2018. Available from: https:// www.fda.gov/news-events/press-announcements/fda-approves-firstgeneric-versions-suboxone-sublingual-film-which-may-increaseaccess-treatment. Accessed December 6, 2019.

6. Office of Generic Drugs. Food and Drug Administration; 2021. Available from: https://www.fda.gov/about-fda/center-drug-evalua tion-and-research-cder/office-generic-drugs. Accessed December 21, 2021.

7. Binder P, Messaadi N, Perault-Pochat M-C, Gagey S, Brabant Y, Ingrand $\mathrm{P}$. Preference for brand-name buprenorphine is related to severity of addiction among outpatients in opioid maintenance treatment. J Addict Dis. 2016;35(2):101-108. doi:10.1080/10550887. 2015.1136494

8. Knopf A. Is generic buprenorphine film as good as Suboxone? Alcohol Drug Abuse Weekly. 2019;31(23):5-6. doi:10.1002/ adaw.32391

9. Datt A, Hirsch AR. 108 warning: generic Suboxone not equal to name brand. CNS Spectr. 2020;25(2):270. doi:10.1017/S10928 52920000267

10. Santiago D, Rosario Y, Melin K, et al. Brand-to-generic substitution of buprenorphine/naloxone sublingual film in Puerto Rico: a case study. P R Health Sci J. 2021;40(4):192-194.

11. Campbell MD, Kolodner G, Spencer RA, DuPont RL. Drug test results as a predictor of retention among patients using buprenorphine in a comprehensive outpatient treatment program. J Addict Dis. 2016;35(4):315-324. doi:10.1080/10550887.2016.1139427

12. Hui D, Weinstein ZM, Cheng DM, et al. Very early disengagement and subsequent re-engagement in primary care Office Based Opioid Treatment (OBOT) with buprenorphine. J Subst Abuse Treat. 2017;79:12-19. doi:10.1016/j.jsat.2017.05.010

13. Vanderkam P, Gagey S, Ingrand P, et al. Are patients' pejorative representations of buprenorphine associated with their level of addiction and of misuse? Drug Alcohol Depend. 2018;188:10-15. doi:10.1016/j.drugalcdep.2018.03.033
Patient Preference and Adherence

\section{Publish your work in this journal}

Patient Preference and Adherence is an international, peer-reviewed, open access journal that focusing on the growing importance of patient preference and adherence throughout the therapeutic continuum. Patient satisfaction, acceptability, quality of life, compliance, persistence and their role in developing new therapeutic modalities and compounds to optimize clinical outcomes for existing disease states are major areas of interest for the journal. This journal has been accepted for indexing on PubMed Central. The manuscript management system is completely online and includes a very quick and fair peer-review system, which is all easy to use. Visit http:// www.dovepress.com/testimonials.php to read real quotes from published authors. 